\title{
Structural changes in molten CdTe
}

\author{
L. Shcherbak ${ }^{1}$, P. Feychuk ${ }^{1}$, Yu. Plevachuk ${ }^{2}$, Ch. Dong ${ }^{3}$ and V. Sklyarchuk ${ }^{2}$ \\ ${ }^{I}$ Chemical Department, University of Chernivtsi, 2, vul. Kotsyubinskogo, 274012 Chernivtsi, Ukraine \\ ${ }^{2}$ Institute of Applied Physics, Ivan Franko National University, 49 General Chuprynka st. 79044 Lviv, Ukraine \\ ${ }^{3}$ Institute of Physics, Technical University Chemnitz, D-09107 Chemnitz, Germany
}

\begin{abstract}
Shear viscosity $(\eta)$ measurements of CdTe and CdTe +2 at $\%$ In melts were performed using a cup viscometer up to $1403 \mathrm{~K}$. The $\mathrm{h}(\mathrm{T})$ dependencies obtained during slow heating and cooling $\left(V_{h / c}=\right.$ $=10-15 \mathrm{~K} / \mathrm{h}$ ) show hysteresys near a melting point. According to the $\eta(T)$ dependencies data drastic changes the melts structure occurred at $1376 \mathrm{~K}$ both during the heating and cooling of the melts.
\end{abstract}

Keywords: CdTe, viscosity, structural changes, melts

Paper received 04.07.00; revised manuscript received 09.10.00; accepted for publication 12.12.00.

\section{Introduction}

In recent years, many efforts have been made to investigate a melt structure and its correlation with growth properties for different substances. Investigations of density, viscosity, electrical resistivity, adiabatic compressibility and other structural-sensitive properties of molten semiconductors lead to the conclusion that a postmelting effect exists both in elements $(\mathrm{Ge}, \mathrm{Si})$ and complex $\left(\mathrm{A}^{3} \mathrm{~B}^{5}\right.$, $\mathrm{A}^{4} \mathrm{~B}^{6}$ etc.) objects [1]. It was shown that nonisothermal gradual phase transition from solid to liquid occurs in certain temperature range above a melting point $\left(T_{m}\right)$ through some steps of crystal-like structural units (clusters) transformation. Therefore, a liquid remains twostructural one in a certain temperature range above $T_{m}$. Nevertheless, it is not clear a priory whether or not the structural transformation occurs isothermally as first order transition or diffusely, as a second order one. For example, rapid structural changes in molten $\mathrm{Si}$, Te-Se, $\mathrm{Ge}-\mathrm{Te}$ and $\mathrm{Cd}-\mathrm{Sb}$ as well as in other systems were revealed by diffraction methods or by heat capacity temperature dependencies $[2,3]$, but their nature remains unknown.

It seems to be of interest to study a transformation of cadmium telluride melt structure with temperature. Previously, thermodynamic analysis of molten CdTe structure was usually performed in the framework of associated solutions model, assuming as the structural units atoms $\mathrm{Cd}$ and Te and associates Cd-Te only. According to [4], a mole fraction of associated species Cd-Te near $T_{m}$ is estimated to be 0.908. Comparing both the calculated and experimental (based on calorimetric study) values of thermodynamic parameters of CdTe fusion, the author of [5] concluded that full entropy was not realized at $T_{m}(\mathrm{CdTe})$. This prediction was illustrated later by DTA method in [6-9] where small additional endothermic effects (AEE) were obtained at 1373, 1379,1393 and $1413 \mathrm{~K}$ at certain thermodynamic conditions, i.e. the postmelting effect in the CdTe melts was revealed. It was proposed in $[8,9]$ that the CdTe melt in the vicinity of $T_{m}$ up to $T_{m}+10 \mathrm{~K}$ can be considered as a solution of crystal-like clusters in the liquid. Upon heating, the clusters undergo thermal dissolution, which may be due to either dissolution from the surface or a fragmentation on smaller species. The most correct treating of the structure transformation in molten CdTe needs direct diffraction experiments at various temperatures. But due to chemical aggressivity of this object, the only neutron diffraction study at $1373 \mathrm{~K}$ was performed up to now by Gaspard et al. [10,11]. Surprise of researchers was connected with the fact that the main parameters of crystal (bond distances, valence angle, coordination number) are kept in the liquid. Each atom was surrounded by a distorted tetrahedron of atoms of other species. It was concluded that this liquid structure is analogous to the Connel-Temkin model of alternate amorphous semiconductors that is rather unexpected in liquid semiconductors.

On the other hand, the data of Gaspard et al. agree with electrical properties of the CdTe melt. According to [8, 9,12], CdTe kept semiconductor properties up to $T_{m}+100 \mathrm{~K}$, at least. Ioffe [13] had explained such type of phase transition of semiconductors by affirmation that covalent bonds are retained above $T_{m}$, and a short-range order of crystalline phase is preserved despite the loss of a long-range order.

Based on results of a direct structural investigation $[10,11]$, an ab initio molecular dynamic simulation of $\mathrm{CdTe}$ at three different temperatures $(800 \mathrm{~K}$ - as supercooled state, 1370 - near $T_{m}$ and $3000 \mathrm{~K}$ - as super- 


\section{Shcherbak et al.: Structural changes in molten CdTe}

heated state) has been performed in [14, 15]. It was found that near $T_{m}$ atoms of tellurium form infinite branched chains. Short and simplified chains are still presented in the supercooled phase. As the temperature increases, the chains break, become shorter and transform into closepacked clusters even in the superheated state.

In order to examine this theoretical model a temperature dependence of viscosity as one of the most structural sensitive properties of a liquid has been studied. As published previously in $[12,16]$, the kinematic viscosity of $\mathrm{CdTe}$ decreases gradually with a temperature increase, but a slope of the temperature dependencies near $T_{m}$ differs twice in both references. In the present work, the dynamic viscosity of CdTe and $\mathrm{CdTe}+2$ at $\%$ In during heating and cooling at quasi-equilibrium conditions with heating/cooling rates of $V_{h / c}=0.33-0.42 \mathrm{~K} / \mathrm{min}$ from $T_{m}$ to $1403 \mathrm{~K}$ was measured.

\section{Experimental}

The shear viscosity was measured using the computer controlled cup viscometer similar to [17]. CdTe samples of stoichiometric composition and $1.10^{20} \mathrm{~cm}^{-3} \mathrm{Te}$ excess and $\mathrm{CdTe}$ +2 at $\%$ In were prepared from pure elements ( $6 \mathrm{~N}$ grade) in evacuated and sealed quartz cups. The melts were homogenized at $T_{m}+30 \mathrm{~K}$ during 12 hours and cooled with a furnace. Then the polycrystal charges were heated in the viscometer from room temperatures up to $1403 \mathrm{~K}$ following by the melt cooling with intermediate holding up to solidification. Doped by 2 at $\%$ In CdTe specimen was heated repeatedly from $T_{\text {room }}$ up to $1403 \mathrm{~K}$ with the holding at $1403 \mathrm{~K}$ during $60 \mathrm{~min}$. The heating/cooling rates $V_{h / c}$ were varied from $0.33 \mathrm{~K} / \mathrm{min}$ to $0.5 \mathrm{~K} / \mathrm{min}$.

\section{Results and discussion}

The data of the CdTe shear viscosity $(\eta)$ changes on heating and cooling combined with holding at $1388 \mathrm{~K}(30 \mathrm{~min})$ and $1378 \mathrm{~K}(30 \mathrm{~min})$ with $V_{h / c}=0.33 \mathrm{~K} / \mathrm{min}$ are presented in Fig. 1. Kinetics of the CdTe melting process is illustrated by Fig. 1a. The viscosity growth above $T_{m}=1363 \mathrm{~K}$ up to $T_{\text {crit. }}=1376 \mathrm{~K}$ reflects the raise of the liquid phase volume fraction in this two-structural system. Taking into account that $T_{\text {crit. }}$ is close to the temperature of first observed in [7-9] AEE, one can conclude about the completion of the crystal-like clusters dissolution process near $1376 \mathrm{~K}$. However, reached at $1376 \mathrm{~K}$ the viscosity value retains almost constant during the next heating up to $1379 \mathrm{~K}$ only, following by occurrence a peak on the $\eta(T)$ dependence in a rather narrow temperature range. So, the melt shows typical for homogeneous, Newtonian liquids features above $1387 \pm 1 \mathrm{~K}$ on the heating and from 1403 up to $1376 \mathrm{~K}$ on cooling. The observed on the heating peak did not reproduced during the melt cooling from $1403 \mathrm{~K}$, in spite of the melt holding at $1388 \mathrm{~K}$ during $30 \mathrm{~min}$. Obviously, the hightemperature melt structure is conserved in the course of cooling up to $T_{\text {crit }}$, were the holding near $T_{\text {crit. }}$ caused the noticeable reduction of the melt viscosity. It means

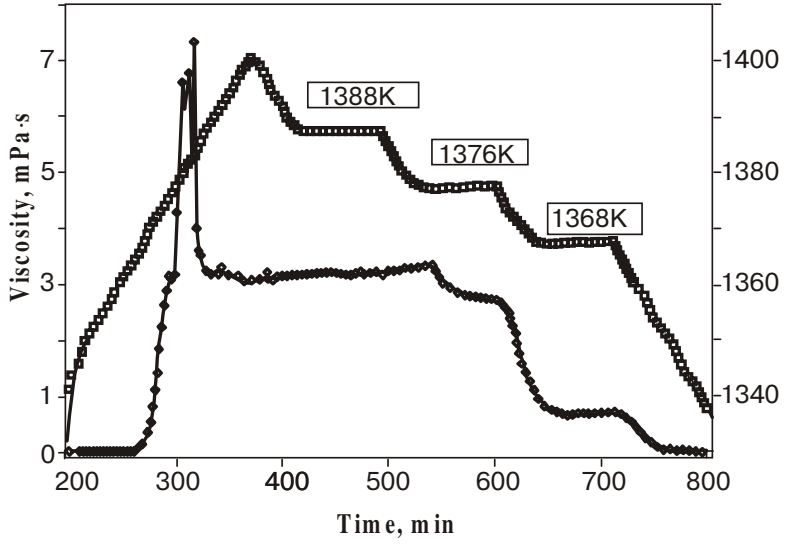

Fig. 1. Temperature dependence of stoichiometric CdTe melt dynamic viscosity. The heating/cooling rate $V_{h / c}=0.33 \mathrm{~K} / \mathrm{min}$.

that structural changes in molten CdTe are not symmetric on heating and cooling at given experimental conditions, except the temperature range near $T_{\text {crit. }}$ demonstrating the hysteresis near $T_{m}$ and above $T_{\text {crit. }}$.

Stability of the melt structure both above $1388 \pm 1 \mathrm{~K}$ up to $1403 \pm 1 \mathrm{~K}$ and below $1403 \pm 1 \mathrm{~K}$ up to $1378 \pm 1 \mathrm{~K}$ is illustrated more clearly by Fig. 1b. One can see that typical for Newtonian liquids viscosity growth with a temperature decreasing is observed in restricted temperature range up to $T_{\text {crit. }}$ only, following by the viscosity reduction.

The viscosity changes on heating and cooling (at $V_{h / c}=$ $=0.42 \mathrm{~K} / \mathrm{min}$ ) of the charge with slight Te excess in comparison with previous case is demonstrated in Fig. 2 a,b. As one can see, a $\lambda$-like view of $\eta(T)$ dependence on heating and critical point where the melt structure changes is reproducible in general. The holding of the cooled melt at $T_{\text {hold }}=1378 \mathrm{~K}$, i.e. the temperature of essential viscosity rise during the melt heating (also does not cause any changes of the melt structure during $30 \mathrm{~min}$, at least. Comparing Figs $1 b$ and $2 b$, one can see that the viscosity of both melts is the same upon cooling, in spite of some difference in composition. The role of the Te excess is

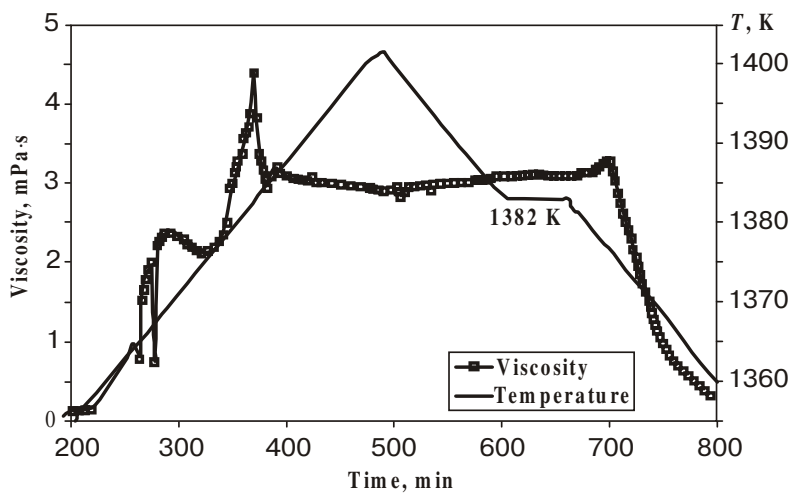

Fig. 2. Temperature dependence of $\mathrm{CdTe}+1.10^{19} \mathrm{at} / \mathrm{cm}^{3} \mathrm{Te}$ melt dynamic viscosity. The heating/cooling rate $V_{h / c}=0.4 \mathrm{~K} / \mathrm{min}$. 


\section{Shcherbak et al.: Structural changes in molten CdTe}

more visible in the fact of the CdTe melting point reduction. Besides, the Te liquid phase presence in solid $\mathrm{CdTe}$ accelerates the clusters dissolving in the melt in comparison with the case of the stoichiometric CdTe, however $T_{\text {crit. }}$ remains the same.

The essential elevation of the heating melt viscosity above the cooling one at the temperature range from 1376 \pm $\pm 1 \mathrm{~K}$ to $1388 \pm 1 \mathrm{~K}$ witnesses about certain intensive chan-ges of the melt structure in the course of heating. The latter resulted in growth of internal friction forces, which compensate and exceeds the action of temperature rise leading to a rise of atom mobility. Taking into account the calculated by Godlevsky et al. $[14,15] \mathrm{CdTe}$ melt structure model, it should be supposed that the Te branched chains formation is the reason of the $\lambda$-like view of $\eta(T)$ dependence of the heating melt, but this process occurs in a rather narrow temperature range. Obviously, the intensive destruction of the chains above the temperature of the top of the peak resulted in creation of some smaller structure units. Taking into account that $\eta(T)$ dependencies of the heating and cooling melt coincide in 1388-1403 K range, one can conclude that a packing of this structure units is the same in whole investigated temperature range up to $T_{\text {crit. }}$.

It must be noted that «anomalous» temperature dependence of viscosity which pass through a maximum at $T_{m}+$ $(60 \div 100) \mathrm{K}$, was obtained in case of other compounds with semiconductor-semiconductor type of the melting, e.g. $\mathrm{Ga}_{2} \mathrm{Te}_{3}$ and $\mathrm{In}_{2} \mathrm{Te}_{3}$ by Tsuchiya et al. $[18,19]$. The authors $[18,19]$ had regarded these melts as superposition of two structures: a loose low-temperature structure which retain the main parameters of solid state, and more densely packed high temperature structure presumably of a linear type (chains and molecules). Really, according to X-ray investigation in [20], a tetrahedral coordination of covalently bonded nearest neighbors remains in molten $\mathrm{In}_{2} \mathrm{Te}_{3}$. It was regarded that tetrahedra are the main structure units up to $T_{m}+30 \mathrm{~K}$, at least, but their packing differs from that in the crystal.

The influence of 2 at $\%$ In on the CdTe viscosity dependence on the temperature was observed during two consecutive heating/cooling processes with $V_{h / c}=0.5 \mathrm{~K} / \mathrm{min}$ (see Fig. 3). The choice of the investigated dopant was connected with some reasons. Thermodynamic analysis of the quasi-binary $\mathrm{CdTe}$ - In system liquidus had revealed close to ideal behavior of the melt components. Besides, it was shown in [8] that the CdTe melt kept its semiconductor nature up to $1473 \mathrm{~K}$ despite the presence of the metallic impurity. Therefore, the short- range order of zinc-blend structure does not change essentially in this two-phase melt. According to [8], the $\mathrm{CdTe}+2$ at $\%$ In melt temperature dependence $\sigma(T)$ had revealed the remarkable changes of the $\sigma(T)$ slope at the temperatures 1373, 1393 and $1413 \mathrm{~K}$, which are close to position of the AEEs in the pure CdTe melt. The most sharp growth of the electrical conductivity of CdTe +2 at \% In melt was observed from 1373 to $1393 \mathrm{~K}$.

As one can see in Fig. 3, similarly to pure CdTe, the doped by In CdTe melt has the hysteresis of the cooling

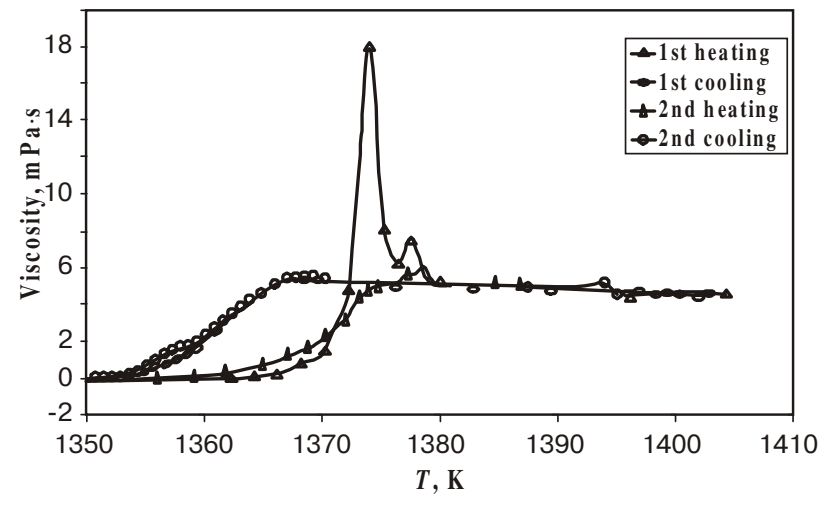

Fig. 3. Temperature dependence of dynamic viscosity for the CdTe +2 at $\%$ In melt $\left(V_{h / c}=0.5 \mathrm{~K} / \mathrm{min}\right)$.

and heating curves both near the melting point and in the vicinity of the peaks on the heating curve. The first peak caused by essential growth of the melt viscosity at $1373 \mathrm{~K}$ following by its rapid decreasing. The second, smaller, peak is observed near $1378 \mathrm{~K}$. The reached above $1380 \mathrm{~K}$ viscosity value, and, consequently, the melt structure became stable as long as the melt is heated up to $1400 \mathrm{~K}$ and then cooled almost to $T_{m}$. Taking into account a similarity of the $\eta(T)$ dependencies of CdTe and $\mathrm{CdTe}+$. In melts, it should be assumed that the first peak in Fig. 3 is caused by Techains formation (according to the theoretical model of $1-$ CdTe transformation by Godlevsky et al $[14,15])$. But break of Te chains during the heating in presence of In atoms occurs in such way that some smaller structural units with the same coordination number, valence angle and interatomic distances, form. Hexagonal Te rings are the most probable candidates for this process. The dissociation of the rings on shorter chains, probably, is eliminated by the second peak at $1379 \mathrm{~K}$ shown in Fig. 3. Note, that the presence of In led to increase of the melt viscosity value (e.g., from approximately $3 \mathrm{mPa} \cdot \mathrm{s}$ in pure CdTe to $5 \mathrm{mPa} \cdot \mathrm{s}$ at $1400 \mathrm{~K})$. According to this version it should be thought that a second order phase transition of the sphalerit - wurtzit type takes place in CdTe and CdTe + + In melts in the vicinity of the peak between 1376 and $1388 \mathrm{~K}$. To maintain this hypothesis, one can add the sharp growth of the $\mathrm{CdTe}+2$ at $\%$ melt electrical conductivity from $1373 \mathrm{~K}$ up to $1393 \mathrm{~K}$, caused, probably, by reorganization of the structural units accompanied with an increase of the broken bonds and configuration entropy changes.

The relaxation of the melt structure during the cooling, obviously, needs much more time in comparison with the process of the crystal structure break by the temperature growth. Using the cooling curves as characteristic of the reached at high temperatures the melts equilibrium state, one can estimate the activation energies of viscous flow $E_{a}$ by the Arrhenius type equation

$\eta(T)=\eta_{\mathrm{o}} \exp \left(-E_{a} / R T\right)$

where $R$ is the universal gas constant. 


\section{Shcherbak et al.: Structural changes in molten CdTe}

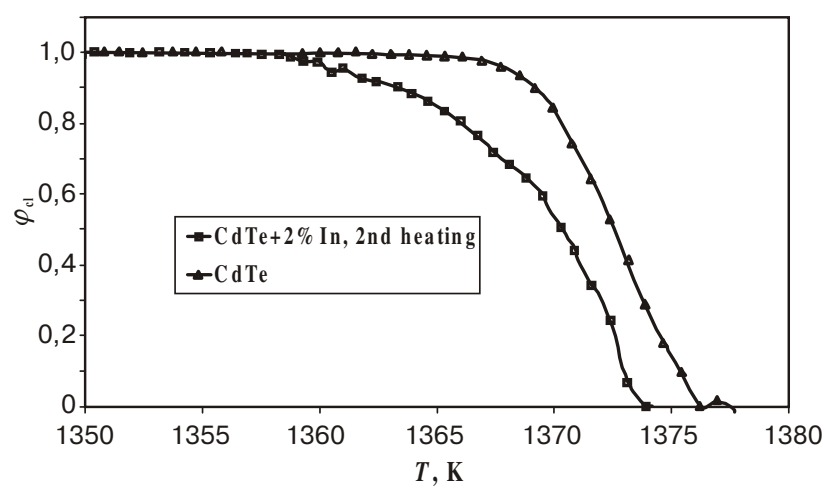

Fig. 4. Volume fraction of clusters in stoichiometric $\mathrm{CdTe}$ and $\mathrm{CdTe}+2$ at $\%$ In melts $v$ s temperature.

The approximation of the viscosity dependencies on temperature by (1) in the temperature ranges $1400 \mathrm{~K}-$ $T_{\text {crit }}$ for CdTe melts (see Figs 1 and 2) and $1400 \mathrm{~K}-T_{m}$ for CdTe +2 at \% In melt (Fig. 3) led us to $E_{a}$ values $51 \pm$ $\pm 3 \mathrm{~kJ} / \mathrm{mole}$ and $66 \pm 1 \mathrm{~kJ} /$ mole for pure and doped melts, accordingly. It is known that $E_{a}$ value is connected with the forces needed for creation of a free volume for replacement of the melt structure unit. $E_{a}$ values obtained using Eq. (1) are close to the typical ones, observed for chemical reactions. Therefore, they show that viscous flow of the investigated melts needs the rupture (and creation) of chemical bonds, retaining in the melts.

The obtained heating curves can be used for an illustration of the crystal- like clusters in content in the melts reduction with the temperature growth. Assuming that the full solving of clusters in the melt occurs when the viscosity value reaches the equilibrium one, we estimate a volume fraction of the clusters dependence on temperature (Fig. 4). As one can see, the presence of the second phase in $\mathrm{CdTe}+\mathrm{In}$ melt intensifies the process of the clusters dissolving, which had finished near $1373 \mathrm{~K}$ in both cases. Therefore, the minimal sizes of created in both melts clusters are close. However, their composition and structure need more detailed investigation.

\section{Conclusions}

The dynamic viscosity measurement vs. temperature $\eta(T)$ is a reasonable tool for studying CdTe melt structure changes. The obtained $\eta(T)$ dependencies on the heating agreed with assumption about postmelting, i.e. nonisothermal CdTe melting process, ranged up to $T_{m}+10=1373 \mathrm{~K}$. The appea-rance of the peak on the $\eta(T)$ dependence between $1376 \mathrm{~K}$ and $1388 \mathrm{~K}$ illuminates certain drastic CdTe melt structure changes, which can be explained both as Te branched chains creation following by their destruction and/or by changes of the tetrahedral structural units type of packing. Similarity of CdTe and CdTe +2 at $\%$ In melts $\eta(T)$ dependencies led to conclusion about similarity of a structurization type for both melts within the same temperature range. According to the viscosity measurements data, the most prominent melt structure rearrange- ment on heating occurs nearby $1388 \pm 1 \mathrm{~K}$. The viscous flow energy activation of coo-led, more equilibrium, CdTe and $\mathrm{CdTe}+2$ at $\%$ In melts was estimated as equal to $51 \pm$ \pm 3 and $66 \pm 1 \mathrm{~kJ} / \mathrm{mole}$, accordingly.

\section{Acknowledgments}

The authors are very grateful to Prof. Suck and Prof. O.E. Panchuk for the initiation of this work. The work is supported in part by European Union Grant «Copernicus» ERBIC 15 CT 960808.

\section{References}

1. V.M. Glasov, Present development of investigation of the postmelting effect in semiconductors melts // Neorgan. Mater. 32 (11), pp. 1287-1305 (1996).

2. Y. Wang and K. Kakimoto, Dislocation effect on crystal-melt interface: an in situ observation on the melting of silicon // J.Cryst.Growth. 208, pp. 303-312 (2000).

3. Y.Tsuchiya.Thermodynamics of structural changes in molten alloys// J.Chem.Phys. 94, pp. 998-1003 (1997).

4. A. Marbeuf, M. Ferah, A. Heutrel, Consistent approach of II-VI equilibrium phase diagrams: application to $\mathrm{Zn}-\mathrm{Se}, \mathrm{Cd}-\mathrm{Te}, \mathrm{Hg}-$ Te and Cd-Hg-Te systems // J. Cryst. Growth. 72, pp. 126-132 (1985).

5. M. Shamsuddin, A. Nazar, Thermodynamic properties of cadmium telluride // High Temp. Sci. 28, pp. 245-254 (1990).

6. P. Rudolph. Fundamental studies of Bridgman growth of CdTe// Prog. Crystal Growth Charact. 29, pp. 275-341 (1994).

7. L.P. Shcherbak, P.I. Feichouk, O.E. Panchouk, Effect of CdTe «postmelting» // J. Crystal Growth. 161, pp. 16-19 (1996).

8. L. Shcherbak, Peculiarities of solid-liquid-phase transition // J. Crystal Growth, 184/185 pp. 1057-1060 (1998).

9. L. Shcherbak, Pre-transition phenomena in CdTe near the melting point // J. Crystal Growth, 197, pp.397-405 (1999)

10. J. Gaspard, C.Bergman, C. Bichara, R. Bellicent, P. Chieux, J. Coffart. Structure of liquid II-VI compounds: CdTe // J. NonCrystalline Solids. 97\&98, pp. 1283-1287 (1987).

11. J. Gaspard, J. Rary, R. Ceolin, and R. Bellissent, Local orders in II-IV liquid compounds// J. Non-Cryst. Solids. 75, pp.205-207 (1996).

12. V.M. Glasov, S.N. Chizhevskaya, N.N. Glagoleva, Liquid Semiconductors, Plenum Press, New York, 1969.

13. A. Joffe and A. Regel, Progress in Semiconductors (Heywood, London, 1960), Vol. 4, p. 237.

14. V.V. Godlevsky, J.J. Derby and J.R. Chelikowsky. Ab initio molecular dynamics simulation of liquid CdTe and Ga As: semicon-ducting versus metallic behavior // Phys. Rev.Lets., 81, pp. 4959-4962 (1998).

15. V.V. Godlevsky, M. Jain, J.J. Derby and J.R. Chelikowsky, Firstprinciples calculations of liquid CdTe at temperatures above and below the melting point // Phys. Rev. B, 60, pp. 86408649 (1999).

16. I.I. Lopatkina and V.P. Bystrov, Vliyanie zameshcheniya rtuti kadmiyem i tsinkom na fiziko-himicheskiye svojstva rasplava HgTe. In: «Khalkogenidy Tsinka, Kadmiya i Rtuti», MISiS N73, Moskva, Metallurgiya, pp. 90-95 (1972).

17. J. Vollman and D. Riedel. The viscosity of liquid Bi-Ga alloys // J .Phys. Condens. Mater. 8, pp.6175-6184 (1996)

18. Y. Tsuchiya, S. Takeda, S. Tamaki, Y. Waseda, E.F.W. Seymour, Evidence for structural inhomogeneity in liquid $\mathrm{In}_{2} \mathrm{Te}_{3} / / \mathrm{J}$. Phys. C 15, pp. 2561-2575 (1982).

19. Y. Tsuchiya, S. Takeda, S. Tamaki, E.F.W. Seymour. Structural inhomogeneity and valence fluctuation in IIIb-Te liquid semiconductors. // J. Phys. C 15, pp. 6447-6512 (1982).

20. Yu.G. Poltavtsev, Struktura poluprovodnikovyh rasplavov. Moskva, Metallurgiya, 1984 (in Russian) 